\title{
Diagnostic Utility of Computerized Tomography in Patients with Orbital Lesions at Tirupati, Andhra Pradesh
}

\author{
Sidda Naik B. ${ }^{1}$, Leelavathamma T. ${ }^{2}$, Lokesh Kumar Y. ${ }^{3}$, Silpa M. ${ }^{4}$ \\ 1, 2, 3,4 Department of Ophthalmology, Sri Venkateswara Medical College, Tirupati, Andhra Pradesh, India.
}

\section{ABSTRACT}

\section{BACKGROUND}

Computer tomography (CT) plays a crucial role in elucidating the etiological diagnosis and identifying the extent of orbital lesions related to thyroid eye disease, cellulitis, orbital osteomyelitis, bone tumours, mucocele, orbital varices and cavernous hemangioma. This study was intended to assess the diagnostic utility of CT in patients presenting with orbital lesions.

\section{METHODS}

We enrolled 30 consecutive patients presenting with orbital lesions, who underwent complete ophthalmological, systemic examination, computerized tomography, and other appropriate systemic investigations over one year period.

\section{RESULTS}

In our clinical cohort of 30 patients, the spectrum of encountered orbital lesions included thyroid eye disease in 8 (26.6\%), orbital fractures in $5(16.6 \%)$, pre-septal hematoma in $3(10 \%)$, cellulitis in $3(10 \%)$, lymphoproliferative lesions in 2 (6.6 $\%)$, orbital lymphomas in 2 (6.6\%), orbital mucocele in $2(6.6 \%)$ cavernous hemangioma in 1 (3.3\%), frontal bone osteomyelitis in 1 (3.3\%), orbital varices in 1 (3.3\%), osteoma in $1(3.3 \%)$ and orbital meningioma in 1 (3.3\%) patients. Proptosis was observed in $22(77 \%)$ patients. On CT imaging, lesion attenuation was hyperdense in $28(92 \%)$ and isodense in $2(8 \%)$ patients. Lesions were extraconal in 19 (63\%), conal in 8 (27\%) and intraconal in $3(10 \%)$ patients. Extraocular muscle involvement was seen in $8(27 \%)$ and bone involvement in 11 (37\%) cases.

\section{CONCLUSIONS}

Computerized tomography in the orbital lesions accurately established the nature and extent of lesions. In $100 \%$ of cases, CT was helpful in deciding appropriate systemic investigations to establish the etiological diagnosis.

\section{KEY WORDS}

Computerized Tomography, Orbital Lesions, Etiological Diagnosis.
Corresponding Author: Dr. Leelavathamma T., Assistant Professor, Department of Ophthalmology, Sri Venkateswara Medical College, SVRR Govt. General Hospital, Tirupati-517501, Andhra Pradesh, India. E-mail: tlmsophthal@gmail.com

DOI: $10.14260 / j e m d s / 2021 / 805$

How to Cite This Article:

Naik SB, Leelavathamma T, Kumar LY, et al. Diagnostic utility of computerized tomography in patients with orbital lesions at Tirupati, Andhra Pradesh. J Evolution Med Dent Sci 2021;10(45):3988-3992, DOI: $10.14260 /$ jemds/2021/805

Submission 02-11-2021, Peer Review 11-11-2021, Acceptance 21-12-2021, Published 29-12-2021.

Copyright (c) 2021 Sidda Naik B. et al. This is an open access article distributed under Creative Commons Attribution License [Attribution 4.0 International (CC BY 4.0)] 


\section{BACKGROUND}

Orbital lesions pose a diagnostic challenge to clinicians given the vast spectrum of the etiologies and complexity of orbital anatomy. Computerized tomography (CT) is simple, noninvasive rapid diagnostic test. Before the advent of CT imaging, invasive explorative orbitotomy was needed to establish an accurate etiological diagnosis. The clarity of presentation of orbital structures by computed tomography has prompted investigations into the detailed multiplanar anatomy of orbit. Tumour margins and inflammatory processes are so clearly defined that one can place the lesion in specific compartments and accurately determine the extent of disease processes. Information regarding the anatomical location of orbital lesions, involvement of intraorbital structure and extension into periorbital regions such as sinuses and intracranial extension can be obtained routinely. Attenuation of the lesions on CT helps in understanding the nature of the lesion and tissue of origin. Multiplanar sequences in CT help in the better anatomical location of the lesion, involvement of orbital structures and extension into peri-orbital regions such as sinuses. ${ }^{1-5}$

\section{Objectives}

To study the diagnostic utility of computerized tomography in defining the cause, nature, and extent of the lesion in clinically diagnosed cases of the orbital lesion.

\section{METHODS}

This prospective, observational study was conducted in the Department of Ophthalmology, SVRR Govt. General Hospital for one year from June 2018 to May 2019. All the patients presenting to the department with orbital lesions during the study period and willing to give consent were included in the study. Pregnant women, claustrophobic, unconscious or incoherent trauma patients and who were not willing to give consent were excluded from the study. This study was approved by the Institutional ethics committee of our institute. Written informed consent was obtained from all the study participants.

The sample size was calculated based on the prevalence of orbital lesions ${ }^{13}(26 \%)$ and found the minimum sample size required as 30 cases. All the patients included in the study underwent detailed clinical ophthalmological, systemic examinations and appropriate systemic investigations. Whenever indicated patients underwent investigations like colour Doppler, ultra-sound B scan, X-ray of Orbits. CT scan (GE Revolution 16 slice CT scanner, USA) was done in all the cases. All the initial diagnosis of CT was confirmed by intraoperative findings. All patients were scanned in a supine position with headfirst in the gantry. Axial sections were taken using Reid's baseline as reference employing $3 \mathrm{~mm}$ sections, later coronal and sagittal reformatting was done in all cases. An appropriate window setting was done to study soft tissue and bone elements. Attenuation of lesions was classified into isodense, hypodense and hyperdense. Hounsfield units (HU) of the lesions were classified as soft tissues if they were between +40 and +80 and bone if the value was between +200 and +1000 . Location of a lesion in relationship with muscular cone was noted as to whether it was intraconal, extraconal or conal. Involvement of extraocular muscles, optic nerve, bones and extension into paranasal sinuses, intracranial spaces was noted. Proptosis was measured objectively by the distance between the anterior and posterior borders of the eyeball and a reference line called the Inter-zygomatic line (IZL).

\section{Statistical Analysis}

All the details were entered into standard proforma, and data was tableted using Microsoft excel. Descriptive statistics were summarized as frequencies with percentages.

\section{RESULTS}

During the above mentioned year, 30 patients fulfilling the inclusion criteria were studied. The age of the patients ranged from 21 to 70 years. A maximum number of patients were in the $4^{\text {th }}$ decade of life (33\%). In gender distribution, there was slight male predominance with 16 (53\%) and 14 (47\%) were females. The most common presenting complaint was isolated proptosis 17 (57 \%), followed by pain and ecchymosis in 8 (27\%), pain and proptosis in 4 (13\%), and pain proptosis and ptosis in 1 (3\%). Unilateral eye involvement was seen in $22(77 \%)$ patients and bilateral was seen in 8 patients. In those patients with unilateral eye involvement, right eye was involved in 11 (36.5\%) and left in $11(36.5 \%)$. A total of 22 (77\%) patients had proptosis and that 11 (36.5\%) had axial proptosis and 11 (36.5\%) had eccentric proptosis.

\section{CT Based Diagnosis of the Orbital Lesion}

Out of 30 patients, the CT based diagnoses were as follows (Figure 1), thyroid eye disease 8 (26.6\%), orbital fractures 5 (16.6\%), pre-septal hematoma $3(10 \%)$, cellulitis $3(10 \%)$, lymphoproliferative lesions 2 (6.6\%), orbital lymphomas 2 (6.6\%) (Figure 2), orbital mucocele 2 (6.6\%) (Figure 3), cavernous hemangioma 1 (3.3\%) (Figure 4), frontal bone osteomyelitis 1 (3.3\%), orbital varices 1 (3.3\%) (Figure 5), osteoma 1 (3.3\%) and orbital meningioma 1 (3.3\%). Table 1 shows the CT based etiological diagnosis of the study populations.

\section{Extension of Lesions}

Out of 30 patients, 19 (63\%) had lesions in the extraconal compartment, 8 (27\%) patients had lesions in a conal compartment and $3(10 \%)$ had lesions in an intraconal compartment. Paranasal sinus involvement was seen in 11 (37\%), extra-ocular involvement was observed in 8 (27 \%). Bone involvement was seen in 11 (37 \%) patients, among them most of them had a fracture of the orbital bones due to fracture. One patient had intra-orbital extension of parasellar meningioma. 
Attenuation of the Lesion

Most of the lesions were hyperdense in 28 (92\%) cases. Isodense lesions were noted in $2(8 \%)$ cases. No hypodense lesion was noted in the present study.

\section{Hounsfield Units of the Lesion}

Out of 30 patients, 24 had lesions corresponding to Hounsfield units (HU) density between +40 and +80 (soft tissue). One patient had lesions corresponding from +200 to $+1000 \mathrm{HU}$.

\begin{tabular}{|c|c|c|}
\hline CT Diagnosis & No. of Patients & Percentage (\%) \\
\hline Thyroid eye disease & 8 & 27 \\
\hline Orbital Fractures & 5 & 17 \\
\hline Pre-Septal Hematoma & 3 & 10 \\
\hline Orbital cellulitis & 3 & 10 \\
\hline Lymphoproliferative Lesions & 2 & 7 \\
\hline Orbital lymphomas & 2 & 7 \\
\hline Orbital mucocele & 2 & 7 \\
\hline Cavernous Hemangioma & 1 & 3 \\
\hline Frontal bone osteomyelitis & 1 & 3 \\
\hline Orbital varices & 1 & 3 \\
\hline Orbital osteoma & 1 & 3 \\
\hline Orbital & 1 & 3 \\
\hline \multicolumn{3}{|c|}{ Table 1. CT Based Diagnosis of the Study Population } \\
\hline Study & Age Range (Years) & Gender Ratio \\
\hline Present study & $21-70$ & $1.1: 1$ \\
\hline B. Usha latha et $\mathrm{al}^{6}$ & $2-70$ & $1.2: 1$ \\
\hline Sharma P et al ${ }^{7}$ & $3-73$ & $2: 1$ \\
\hline KK Sabharwal et al ${ }^{8}$ & $2-80$ & $1.08: 1$ \\
\hline Table 2. Comparis & $\begin{array}{c}\text { of Studies Accordi } \\
\text { and Gender Ratio }\end{array}$ & o the Age Range \\
\hline
\end{tabular}

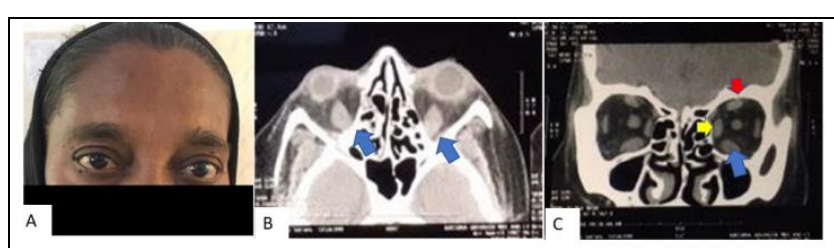

Figure 1. (A) Patient with Thyroid Eye Disease. (B) CT Orbit Axial Sections Showing Inferior Rectus Muscle Enlargement. (C) CT Orbit Coronal Section Showing Enlargement of the Inferior rectus (Blue Arrow), Medial Rectus (Yellow Arrow) and Superior Rectus (Red Arrow)

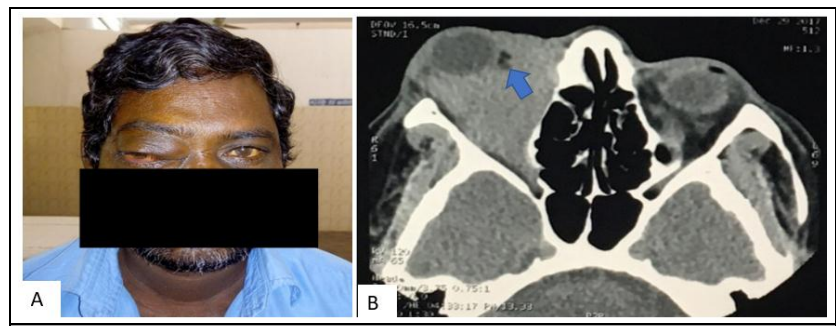

Figure 2. (A) Patient with Eccentric Proptosis of the Right Eye. (B) CT Orbit Axial Sections Showing Well Defined Homogenous Hyperdense Lesion with Localized Hypodensity (Blue Arrow) Displacing the Globe Laterally, Eroding the Medial Wall of Orbit Suggestive of Orbital Lymphoma

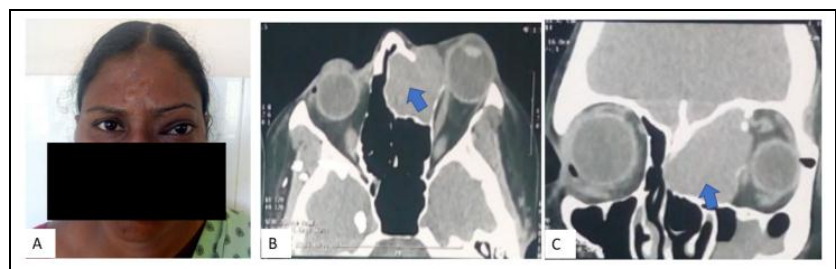

Figure 3. (A) Patient with Eccentric Proptosis of the Left Eye. (B) CT Orbit Axial Sections Show Well Defined Homogenous Hyperdense Lesion in the Frontal Extending Medially (Blue Arrow) Displacing the Globe Laterally, Eroding the Medial Wall of Orbit Suggestive of Orbital Mucocele. (C) CT Orbit Coronal Section Showing Opacification of the Frontal Sinus (Blue Arrow), Eroding the Medial Wall of the Orbit and Extending to the Orbit with Lateral Displacement of the Globe

\begin{tabular}{|c|c|c|}
\hline Study & Most Common Aetiology & Percentage (\%) \\
\hline Present study & Thyroid eye disease & 27 \\
\hline Sharma P et al ${ }^{7}$ & Pseudotumor & 30 \\
\hline Vikas Agarwal et al $^{9}$ & Mass lesion & 46.87 \\
\hline K K Sabharwal et al ${ }^{8}$ & Tumors & 46 \\
\hline Pushpa Ranjan et al ${ }^{10}$ & Malignant lesions & 29.72 \\
\hline \multicolumn{3}{|c|}{$\begin{array}{l}\text { Table 3. Comparison of Most Common Etiologies } \\
\text { Found in Other Studies }\end{array}$} \\
\hline
\end{tabular}
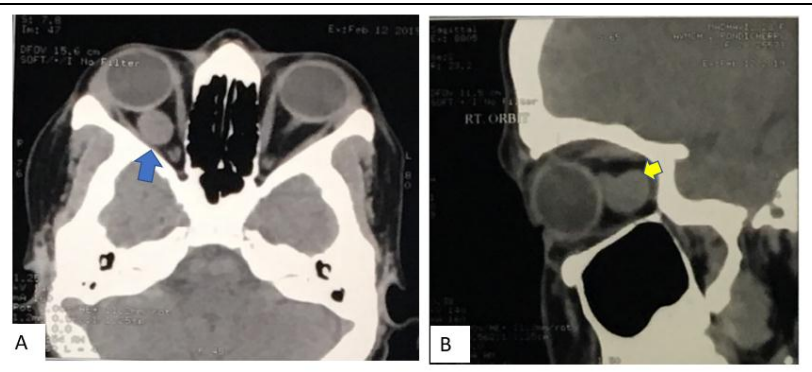

Figure 4. (A) CT Orbit Axial Sections Showing Well Defined Homogenous Oval Mass Slightly Hyperdense to the Muscles. (B) CT Orbit Sagittal Section Showing Well Defined Oval Mass without Calcification Seen Separately from the Globe and Orbit Suggestive of Cavernous Hemangiomas

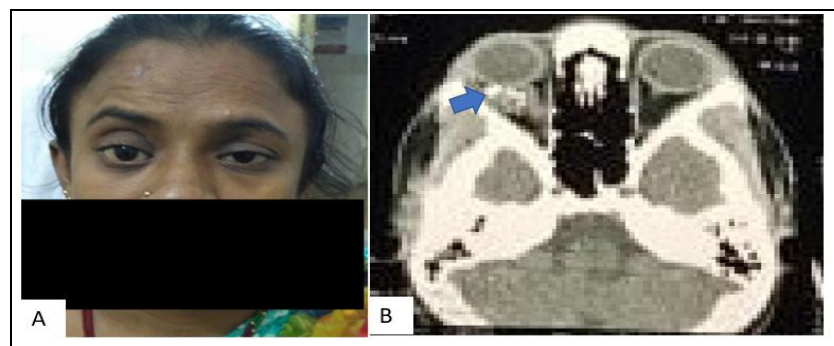

Figure 5. (A) Patient with Right Eye Axial Proptosis. (B) CT Orbit Axial Sections Showing Irregular Heterogeneously Hyperdense Club-Shaped Soft Tissue Mass with Tapering towards the Orbital Apex (Blue Arrow) Suggestive of Phleboliths Seen in Congenital Orbital Varices

\section{DISCUSSION}

The strength of CT orbits includes exquisite bone details, speed of examination and excellent spatial resolution. Apart from assessing the nature and extent of the lesion, the CT guides in taking the biopsy from the lesions. Our age and gender ratio were comparable with the previously done studies (Table 2).

A total of 12 types of orbital lesions were diagnosed in 30 cases with thyroid eye disease being the most common lesion in $27 \%$ of patients. Our findings were comparable with a study done by Vikas Agarwal et al. Bilateral symmetric fusiform enlargement of the multiple extra-ocular muscles with sparing of the tendon insertion was the characteristic finding observed in all cases of thyroid eye disease. A comparison of the most common etiologies found in other studies is shown in table 3 .

The most common presenting clinical feature in the present study was proptosis. Vikas Agrawal et al. ${ }^{5}$ in their study, found pain/headache $(62.50 \%)$ to be the most common presentation in contrast to the present study. Mohammed Ismail et al. in their study of 50 cases with a clinically suspected orbital space-occupying lesion, observed that the most common presenting feature was proptosis $(62$ $\%)$ followed by swelling (46\%). The results of this study correlated well with the present study. Orbital fractures were noted in $17 \%$ of patients. Our results are comparable to the 
study done by EA Parthasarathy et al. Presepatal hematoma was seen in $10 \%$ of the patients.

Orbital cellulitis was observed in three patients (10\%) in the present study. On CT examination, there was diffuse soft tissue thickening of the eyelid [pre-septal compartment] with the extension of inflammation into post-septal space. The extraconal lesion was hyperdense with Hounsfield units 42, 42 and $45 \mathrm{HU}$ corresponding to soft tissue. Extraocular muscles, optic nerve and the superior ophthalmic vein were normal. Ethmoidal sinusitis was noted in all cases. There was no evidence of any bony erosion. Two patients were diagnosed with a lymphoproliferative lesion (7 \%). They had a homogenous soft tissue lesion with 45-50 HU attenuation in the extraconal compartment. HPE confirmed the diagnosis of non-Hodgkin's lymphoma. Two patients (7 \%) had homogenous slightly hyperdense lesions involving intraconal and extraconal compartments. HPE showed Hodgkin's lymphoma. Our findings were comparable with previously done studies. ${ }^{6-11}$

Orbital mucocele was found in 2 patients $(7 \%)$. CT examination showed completely opacified frontal, ethmoidal and sphenoidal sinuses with a homogenous hyperdense mass lesion with thinning of the sinus wall. Areas of bone resorption were noted with bony defect and extension of mass into adjacent orbit. A CT diagnosis of frontoethmoidal and sphenoidal mucocele was made which was confirmed by an ENT surgeon. One patient had orbital varices. KK Sabharwal et al. ${ }^{4}$ observed a similar proportion of patients in their study. One patient had an orbital meningioma. There was an evidence of stippled calcification noted along the optic nerve. The intracranial extension was noted up to optic chiasma. One patient was diagnosed with orbital osteoma based on the CT findings of an ill-defined hyperostotic lesion (532 HU corresponding to bone) extending from the ethmoidal sinus into the orbit. The present study correlated with the studies done by B. Ushalatha et al. ${ }^{6}$ and $\mathrm{KK}$ Sabharwal et al. ${ }^{8}$

\section{Compartment Involvement}

The extraconal compartment was involved in 19 patients (63 $\%$ ) which was mostly due to orbital non-Hodgkin's' lymphoma. Conal compartment was involved in 8 patients (27 \%) who were all due to thyroid eye disease. The intraconal compartment was involved in 3 patients $(10 \%)$ that were due to orbital cavernous haemangioma, orbital meningioma and orbital varices. In a study done by $\mathrm{B}$. Ushalatha et al. ${ }^{6}$ multi-compartmental lesions were the most common types observed in $44 \%$ followed by $27 \%$ of extraconal lesions and most of them were due to haemangiomas. In a study done by Mohammed Ismail et al., 12 extraconal orbital lesions were found in $60 \%$ of cases and intraconal lesions in $22 \%$ of cases and $10 \%$ of cases involved both. This study correlated with the present study as in our study, extraconal lesions were the most common.

\section{Hounsfield Units}

Hounsfield unit of +40 to +80 was seen in 24 patients that corresponded to soft tissue and most of these cases were due to thyroid eye disease. Hounsfield unit of +200 to +1000 was seen in one case which was due to orbital osteoma (532 HU).
CT scan is superior to MRI in cases that require visualization of bone (e.g.: trauma, hyperostosis, PNS disease) and in cases of metallic foreign bodies in the orbit (where MRI is contraindicated). CT scan of orbits is the imaging modality of choice in ocular trauma due to RTA (Road Traffic Accidents) where MRI imaging cannot be performed on an emergency basis.

\section{CONCLUSIONS}

CT is a preferred investigational modality in assessing orbital lesions. Multiplanar sections (axial, coronal and sagittal sections) improve diagnostic accuracy. Choosing the appropriate investigation in each clinical situation enhances the outcome. Maximum information can be obtained by appropriate scanning technique and systematic analysis of CT characters of various lesions involving the orbit. Any extraocular extension or intracranial extension helps in determining prognosis. Compartmentalization helps in arriving at a diagnosis. CT is fairly accurate in narrowing the differential diagnosis.

\section{Limitations}

The sample size was relatively small. Follow up of all cases was not done. Correlation with MRI was done in certain special situations. The study duration was short.

Data sharing statement provided by the authors is available with the full text of this article at jemds.com.

Financial or other competing interests: None.

Disclosure forms provided by the authors are available with the full text of this article at jemds.com.

\section{REFERENCES}

[1] Dubey RB, Tara NP, Sisodiya KN. Computerised tomographic evaluation of orbital lesions: Pictorial essay. Indian J Radiol Imaging 2003;13:261-70.

[2] Kalender WA, Seissler W, Klotz E, et al. Spiral volumetric CT with single-breath-hold technique, continuous transport, and continuous scanner rotation. Radiology 1990;176(1):181-3.

[3] Fuchs T, Kachelriess M, Kalender WA. Technical advances in multi-slice spiral CT. Eur J Radiol 2000;36(2):69-73.

[4] Flohr TG, Schaller S, Stierstorfer K, et al. Multi-detector row CT systems and image-reconstruction techniques. Radiology 2005;235(3):756-73.

[5] Lee AG, Brazis PW, Garrity JA, et al. Imaging for neuroophthalmic and orbital disease. Am J Ophthalmol 2004;138(5):852-62.

[6] Ushalatha B, Sambasivarao K. Role of CT in the evaluation of orbital tumors. IOSR-JDMS 2016;15(4):2279.

[7] Sharma P, Tiwari PK, Ghimire PG, et al. Role of computed tomography in evaluation of proptosis. Nepal J Med Sci 2013;2(1).34-7.

[8] Sabharwal KK, Chouhan AL, Jain S. CT evaluation of proptosis. Indian J Radiol Imaging 2006;16(4):683-8. 
[9] Agrawal V, Madan M, Jajodia S, et al. Evaluation of proptosis by using computed tomography in a tertiary care center, Burla, Sambalpur, Odisha. J Evid Based Med Healthc 2017;4(56):3397-400.

[10] Ranjan P, Ranjan R. CT evaluation of diseases of orbit. J Evolution Med Dent Sci 2016;5(47):2993-9.

[11] Parthasarathy EA, Einstien A, Sulaiman FA, et al. Computed tomography in orbital pathology with clinicopathological correlation. Int J Contemporary Med Surg Radiol 2018;3(2):B60-4.

[12] Ismail M, Priyanka B, Shashikumar MR, et al. Role of computed tomography in the assessment of the orbital space occupying lesions. Int J Contemporary Med Surg Radiol 2018;3(4):D90-4. 